\title{
EFFECT OF CHANGES IN CYCLE ERGOMETER SETTINGS ON BIOELECTRICAL ACTIVITY IN SELECTED MUSCLES OF THE LOWER LIMBS
}

\author{
ROBERT STASZKIEWICZIA, MICHAŁ KAWULAKIA, LESZEK NOSIADEKIA, JAROSŁAW OMORCZYKIB, \\ ANDRZEJ NOSIADEK ${ }^{2}$ \\ ${ }^{1}$ University of Physical Education in Krakow, Faculty of Physical Education and Sport, \\ Department of Biomechanics ${ }^{a}$, Department of Gymnastics and Dance ${ }^{b}$ \\ 2State Higher Vocational School in Tarnow, Department of Physical Education
}

\author{
Mailing address: Robert Staszkiewicz, University of Physical Education in Krakow, Faculty of Physical Education \\ and Sport, Department of Biomechanics, 78 Jana Pawla II Ave., 31-573 Krakow, tel.: +48 12 6831539, \\ fax: +48 12 6831121, e-mail: robert.staszkiewicz@gmail.com
}

\begin{abstract}
Introduction. The aim of this study was to measure the duration of biopotentials in selected muscles of the lower limbs, evaluate the time of elevated bioelectrical activity in these muscles, and identify similarities and differences in electrical phenomena that occur in the muscles for various external settings of a cycle ergometer. Material and methods. The study examined 10 healthy people ( 5 women and 5 men) aged from 20 to 30 years. A cycle ergometer and EMG apparatus were used in the experiment. The bioelectrical activity of six muscles of the lower limbs (rectus femoris, vastus medialis, tibialis anterior, biceps femoris, gastrocnemius caput mediale, and gastrocnemius caput laterale) was recorded for four different settings of the cycle ergometer (variable saddle height and method of foot attachment to pedals). The EMG records were presented with reference to the bicycle crankset rotation cycle. Conclusions. The study found that changing the height of the saddle of the cycle ergometer and the use of toe clips in the pedals caused changes in bioelectrical activity in the muscles. The adjustment of saddle height affected the duration of potentials more noticeably than the use of toe clips. Furthermore, only one period of elevated electrical activity in the muscles of the lower limbs was found in the pedalling cycle. The longest time of the presence of action potentials was recorded for the $\mathrm{m}$. gastrocnemius caput laterale, whereas the shortest time was observed in the $\mathrm{m}$. vastus medialis.
\end{abstract}

Key words: biomechanics, electromyography, pedalling, muscle, lower limb

\section{Introduction}

Cycling, including riding a stationary bicycle (or cycle ergometer), is a popular topic of scientific research, particularly among researchers in the field of biomechanics [1]. The research typically focuses on such aspects as improved pedalling efficiency [2], energy expenditure for this movement, improved energy expenditure for the movement, and improved cycling comfort [3]. Studies have examined optimal positions on a bike [4, 5] and the choice of adequate and optimal pedalling rate [6].

Scientific interests are also focused on issues related to coordination [7] during the driving motion of the cyclist's body. Some studies have been oriented at the use of new technological solutions in bicycles, ranging from changes in the area of the cogset [8] to the types of foot fixation to the pedals [9].

Numerous biomechanical examinations of locomotor activity on a bicycle have helped gain knowledge useful for physiotherapists. Drozdek et al. [10] evaluated the effect of body position adopted on a bicycle on the recordings of bioelectrical muscle activity (EMG). Similarly, the results of the studies that examined the effect of various pedalling techniques on biomechanical motion indices [11] can have some practical implications.

The interest of physical therapists in the problems discussed results directly from the fact that one of the standard pieces of equipment at surgeries is the stationary bicycle (cycle ergometer). The popularity of this device stems from ease of use, easy control of progress, and uncomplicated load modulation. Furthermore, most patients have their own traditional bikes which can later be used to continue the rehabilitation. Therefore, researchers in biomechanics cooperate with physical therapists to prevent the adverse consequences of cycling $[12,13,14]$, examine the effect of saddle height on knee joint load [15], and improve rehabilitation protocols [16].

This study addresses a combination of problems which have been discussed over the years. The innovative character of the study, however, lies in the fact that it approaches these issues in the context of physiotherapy.

\section{The aim of the study}

As mentioned before, physical therapies are often focused on the patient's movements. Therefore, physiotherapists use various devices and accessories which allow for the engagement of selected muscles, i.e. those that are expected to be activated in the rehabilitation process. One of the ways of stimulating the muscles of the lower limbs is having the patient perform exercise on a cycle ergometer.

The aims of this study were as follows:

a) to measure the duration of the biopotentials of selected muscles of the lower limbs, 
b) to evaluate the time of elevated bioelectrical activity in these muscles,

c) to evaluate the number of such phenomena during the bicycle crankset motion,

d) to describe the sequence of electrical phenomena with reference to specific muscles during pedalling,

e) to explore similarities and differences in electrical phenomena in the muscles for different external settings of a bicycle.

\section{Material and methods}

\section{Research material}

The examinations discussed in this study were carried out in the Department of Biomechanics of the University of Physical Education in Krakow. The study examined 10 healthy people ( 5 women and 5 men) aged from 20 to 30 years. Two exclusion criteria were used during recruitment for the study: involvement in professional sport (cycling) and previous serious injuries to the locomotor system. The study was aimed at identifying a natural pedalling pattern in healthy people and evaluating the effect of external bicycle settings (saddle height and the use of toe clips) on this pattern.

Basic morphological indices, that is body height and body mass, in women were $167.2 \pm 4.4 \mathrm{~cm}$ and $58.1 \pm 9.5 \mathrm{~kg}$ (mean \pm $\mathrm{SD})$. In the male group, these values were $180.7 \pm 7.5 \mathrm{~cm}$ and $75.2 \pm 7.4 \mathrm{~kg}$, respectively.

During the measurement, the study participants wore clothes that did not limit the range of movement, with the most important elements being comfortable shoes and shorts. Only this type of clothes made it possible to glue the electrodes to the muscles, made exercising on the cycloergometer comfortable, and minimised the risk of disturbance in the signal flow from the electrodes to the central station.

\section{Research procedure}

The most important components of the measurement system used in the study were a cycle ergometer (Monark 818 E Ergomedic) and a wireless EMG apparatus (WBA Mega Electronics). The set-up of the electrodes and the wireless signal receivers during the measurement is presented in Figure 1. The basic task

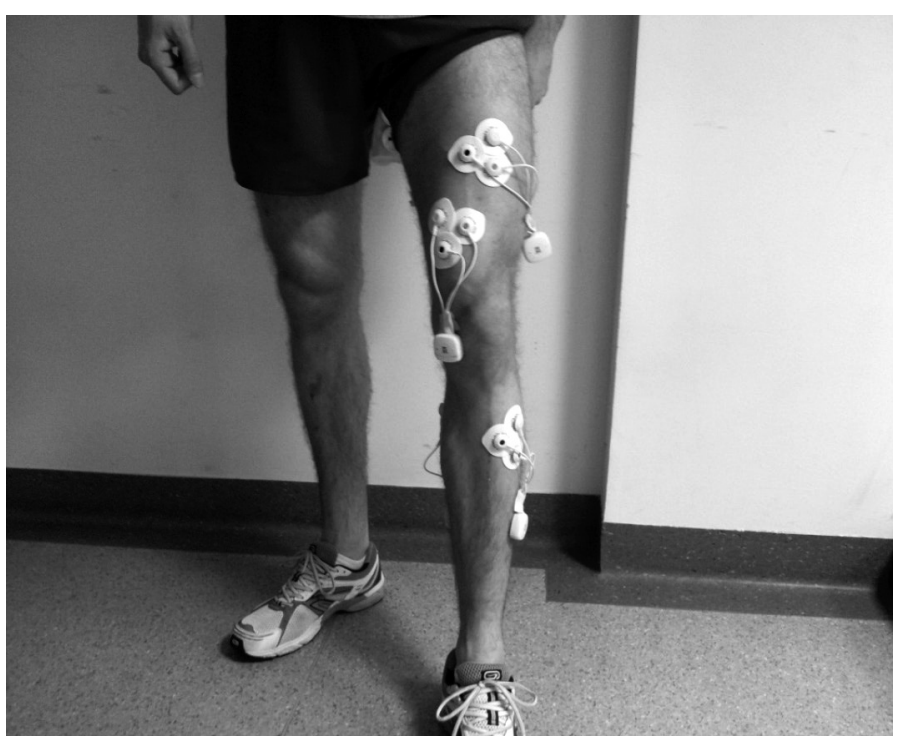

Figure 1. Lower limb with electrodes and wireless signal receivers was to record bioelectrical activity in six selected muscles of one (left) lower limb. The EMG examinations were performed for the following muscles: rectus femoris (RF), vastus medialis (VM), tibialis anterior (TA), biceps femoris (BF), gastrocnemius caput mediale (GCM), and gastrocnemius caput laterale (GCL). Three electrodes were glued to the skin over each muscle (positive, negative, and referential electrodes). Dedicated disposable electrodes were used. The locations of gluing were consistent with the guidelines of the manufacturer of the EMG system and determined with high precision by means of MegaWin computer software.

The hair in the areas where the electrodes which received the electrical potentials from the muscles were glued was shaved using an electric shaver, and the stratum corneum was removed with cotton swabs and spirit. After the electrodes were mounted, the wireless signal receivers were attached. The receivers sent signals to the central station with information about the electrical phenomena in the muscles. Next, the information was processed and displayed on the computer screen.

The examinations of biopotentials were divided into four stages representing four different variants of settings. The first stage consisted in recording them during pedalling with the saddle set high and using bicycle toe clips. Saddle height was adjusted individually so that in the middle of the cycle (when the foot was in the lowest position), the leg was fully extended in the knee joint. The second stage was similar to the first one (the same saddle height), but the toe clips were removed during pedalling. During the third stage, the saddle was lowered each time by half of the height of the first stage, and the toe clips remained detached. In the last (fourth) stage, the toe clips were attached again, and the saddle remained at the lowered height.

In each experiment (stages 1 to 4 ), the study participants pedalled at a constant pedalling rate (rhythm) of $60 \mathrm{rpm}(1 \mathrm{~Hz})$. The participants were allowed to control the pedalling rate in two ways: by watching a display on the cycle ergometer and by listening to the sound from a metronome. Following the guidelines, the starting point of individual pedalling cycles adopted was the highest position of the left pedal.

The biopotentials of the muscles were recorded during their activity for each bicycle setting over a minimum of 15 complete cycles (crankset rotations). The analysis was based on biopotentials recorded for only 10 (consecutive) cycles. The beginning of each cycle was also denoted with a marker in an electronic form by the person who supervised the experiment.

Apart from the constant rotational speed of the crankset (and, consequently, the frequency), constant load was also used at each stage of the experiment. The option designed by the manufacturer of the cycle ergometer was used for this purpose. The load was adjusted each time, using the external setting, so that the mechanical power developed by the muscles of the lower limb was constant for each frequency (100 W).

The duration of the bioelectrical activity in the muscles studied was evaluated for each variant of movement (1-4), the total time of elevated activity was measured, and the number of the occurrences of elevated activity was counted.

\section{Statistical data analysis}

All statistical calculations were performed by means of the Statistica 12 (StatSoft) software. The software was used to perform a descriptive characterisation and an analysis of differences in muscle action potentials for each variant of the cycle ergometer settings (univariate repeated measures ANOVA). 


\section{Results}

The methodology used in the study allowed for the determination of the number of periods during which electrical action potentials in the muscles were recorded during pedalling. One such period was usually recorded for each combination of bicycle settings (variants 1 to 4 ). At the same time, one phase of elevated bioelectrical activity of the muscles was also observed in this period.

The analysis of the total duration of action potentials in the lower limbs demonstrated that in the first variant of the bicycle settings, they lasted on average from $0.47 \mathrm{~s}$ to $0.62 \mathrm{~s}$. In other settings, this time was $0.48-0.68 \mathrm{~s}$ (variant 1 ), $0.48-0.61 \mathrm{~s}$ (variant 3), and $0.49-0.60 \mathrm{~s}$ (variant 4 ). The above ranges of values show that the differences in the level of the variable discussed in the study were minimal. The differences were noticeable when electrical signals from the same muscles were compared for different variants of bicycle settings. The relative time of electrical excitation over a crankset rotation cycle is presented in Table 1.

The data contained in the table reveal that the changes in the height of the saddle had a greater effect on the duration of action potentials in the muscles studied compared to the use of toe clips. Furthermore, the use of toe clips tended to affect the duration of biopotentials more when the saddle was set high than when it was set low. As can be observed, changes in external bicycle settings did not have an effect on the relative duration of VM muscle potentials, which was active approximately $50 \%$ of the cycle time. These settings were found to have the greatest effect on the duration of TA muscle potentials (the action potential recorded for this muscle occurred from $47 \%$ of the duration of pedal rotation, Tc, to $62 \%$ of Tc). However, the univariate repeated measures ANOVA did not reveal a significant effect of different cycle ergometer settings on the duration of action potentials in the muscles. The value of the calculated F statistic did not exceed level 1. Furthermore, the evaluation of the effect size (change in settings) demonstrated that only $13 \%$ of the variation in the results could be attributed to the type of movement task performed (partial eta squared, $\eta 2 \mathrm{P}=0.13$ ).

Table 1. The relative duration of action potential in the muscles of the lower limbs (stages 1-4 - pedalling variants, Tc - duration of pedal rotation)

\begin{tabular}{|c|c|c|c|c|}
\hline & Stage 1 & Stage 2 & Stage 3 & Stage 4 \\
\cline { 2 - 5 } & $\overline{\mathrm{X}} \pm \mathrm{SD}$ & $\overline{\mathrm{X}} \pm \mathrm{SD}$ & $\overline{\mathrm{X}} \pm \mathrm{SD}$ & $\overline{\mathrm{X}} \pm \mathrm{SD}$ \\
\cline { 2 - 5 } & {$[\% \mathrm{Tc}]$} & {$[\% \mathrm{Tc}]$} & {$[\% \mathrm{Tc}]$} & {$[\% \mathrm{Tc}]$} \\
\hline RF & $53.4 \pm 4.2$ & $51.4 \pm 5.4$ & $54.8 \pm 4.8$ & $57.4 \pm 7.8$ \\
\hline VM & $47.5 \pm 3.0$ & $48.3 \pm 3.6$ & $49.1 \pm 5.1$ & $48.8 \pm 5.6$ \\
\hline TA & $47.5 \pm 11.9$ & $62.0 \pm 10.4$ & $48.3 \pm 1.9$ & $53.2 \pm 2.6$ \\
\hline BF & $60.0 \pm 14.5$ & $62.3 \pm 17.8$ & $53.2 \pm 1.9$ & $57.2 \pm 1.8$ \\
\hline GCL & $61.9 \pm 12.1$ & $67.6 \pm 13.7$ & $61.5 \pm 16.7$ & $59.3 \pm 14.9$ \\
\hline GCM & $57.1 \pm 7.9$ & $55.2 \pm 1.1$ & $61.4 \pm 16.7$ & $59.7 \pm 17.5$ \\
\hline
\end{tabular}

As mentioned before, one period of elevated bioelectrical activity in the muscles was recorded for each bicycle setting. Its mean duration is presented in Table 2 . The values contained in the table lead to the conclusion that the time of elevated activity for bicycle settings with the saddle set low (3 and 4) was slightly shorter than for setting variants 1 and 2 (saddle set high). Furthermore, no unequivocal direction of changes in the time of elevated potentials was found in the muscles studied. The univariate repeated measures ANOVA did not demonstrate significant differences in elevated EMG signal during propulsive movements for various cycle ergometer settings.

Table 2. Duration of elevated action potential in the muscles of the lower limbs (stages 1-4 - pedalling variants)

\begin{tabular}{|c|c|c|c|c|}
\hline & Stage 1 & Stage 2 & Stage 3 & Stage 4 \\
\cline { 2 - 5 } & $\overline{\mathrm{X}} \pm \mathrm{SD}$ & $\overline{\mathrm{X}} \pm \mathrm{SD}$ & $\overline{\mathrm{x}} \pm \mathrm{SD}$ & $\overline{\mathrm{x}} \pm \mathrm{SD}$ \\
\cline { 2 - 5 } & {$[\mathbf{s}]$} & {$[\mathbf{s}]$} & {$[\mathbf{s}]$} & {$[\mathbf{s}]$} \\
\hline RF & $0.35 \pm 0.04$ & $0.32 \pm 0.06$ & $0.34 \pm 0.05$ & $0.33 \pm 0.06$ \\
\hline VM & $0.39 \pm 0.04$ & $0.40 \pm 0,04$ & $0.38 \pm 0.06$ & $0.37 \pm 0.06$ \\
\hline TA & $0.27 \pm 0.16$ & $0.26 \pm 0.17$ & $0.22 \pm 0.10$ & $0.32 \pm 0.18$ \\
\hline BF & $0.40 \pm 0.11$ & $0.37 \pm 0.14$ & $0.28 \pm 0.08$ & $0.31 \pm 0.12$ \\
\hline GCL & $0.43 \pm 0.13$ & $0.44 \pm 0.14$ & $0.37 \pm 0.11$ & $0.38 \pm 0.12$ \\
\hline GCM & $0.34 \pm 0.13$ & $0.6 \pm 0.12$ & $0.37 \pm 0.24$ & $0.37 \pm 0.26$ \\
\hline
\end{tabular}

Figures 2-5 illustrate the bioelectrical activity of the muscles for each variant of bicycle settings (1-4). They help determine the sequence of electrical phenomena with reference to specific muscles during pedalling. It is also possible to evaluate the temporal profile of biopotentials (including elevated potentials) and find similarities and differences in electrical phenomena for various bicycle settings.

In the first variant of settings (see Figure 2), electrical phenomena in the muscles studied were observed typically from approximately $10 \%$ of the duration of the pedalling cycle (TA and RF). Electrical excitation started in other muscles when the crankset was located at around $45^{\circ}$. Electrical excitation disap-

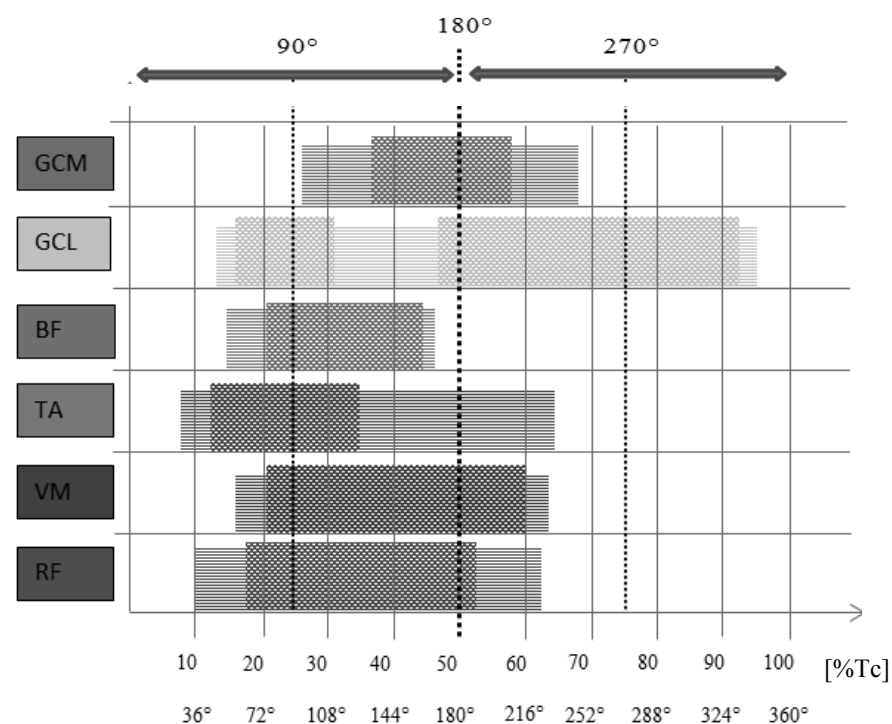

elevated electrical activity

Figure 2. Bioelectrical activity of muscles in the group studied for the first variant of bicycle settings 
peared the earliest in the biceps femoris muscle (before half of the cycle was completed) and the latest in the gastrocnemius caput laterale ( $95 \%$ of the cycle). In other muscles, electrical signals became noticeably weaker at about $65 \%$ of the cycle time ( $225^{\circ}$ of crankset rotation).

The analysis of the figure also reveals that in variant 1 of the motion, two periods of elevated electrical activity were recorded only for the gastrocnemius caput laterale: the first for the angle of rotation of $90^{\circ}$ and the second after half of the cycle was completed. Furthermore, the elevated signals of electrical activity were recorded in the vastus medialis muscle over nearly the entire period.

Figure 3 illustrates bioelectrical activity in the muscles of the lower limbs for the second variant of motion. It can be observed that the detachment of the toe clips from the pedals changed the pattern of the electrical phenomena. The EMG signal was found the earliest in the GCL and TA muscles (approximately $10 \%$ of $\mathrm{Tc}$ ) and the latest in the GCM muscle (approximately $20 \%$ of Tc). Furthermore, action potentials were found in all the muscles until $60-70 \%$ of cycle duration, and the electrical activity was recorded in the GCL and BF muscles nearly until the completion of the crankset motion.

It is noticeable that the signals were recorded the longest for the GCL and BF muscles, but it can also be observed that the former was characterised by two periods of elevated bioelectrical activity separated with a short period of electrical silence.

The bioelectrical activity of the muscles during pedalling on the cycle ergometer with the saddle set low and without toe clips is presented in Figure 4. Except for the tibialis anterior muscle (TA), electrical phenomena in other muscles were recorded after $10 \%$ of cycle duration. The EMG signals in this muscle (TA) disappeared the earliest (at approximately $50 \%$ of cycle duration).

In variant 3 of the settings, the electrical signal was recorded the longest in the GCL muscle, but only one short period of elevated electrical activity was observed (30-40\% of cycle duration). It can be observed that, at around half of the range of

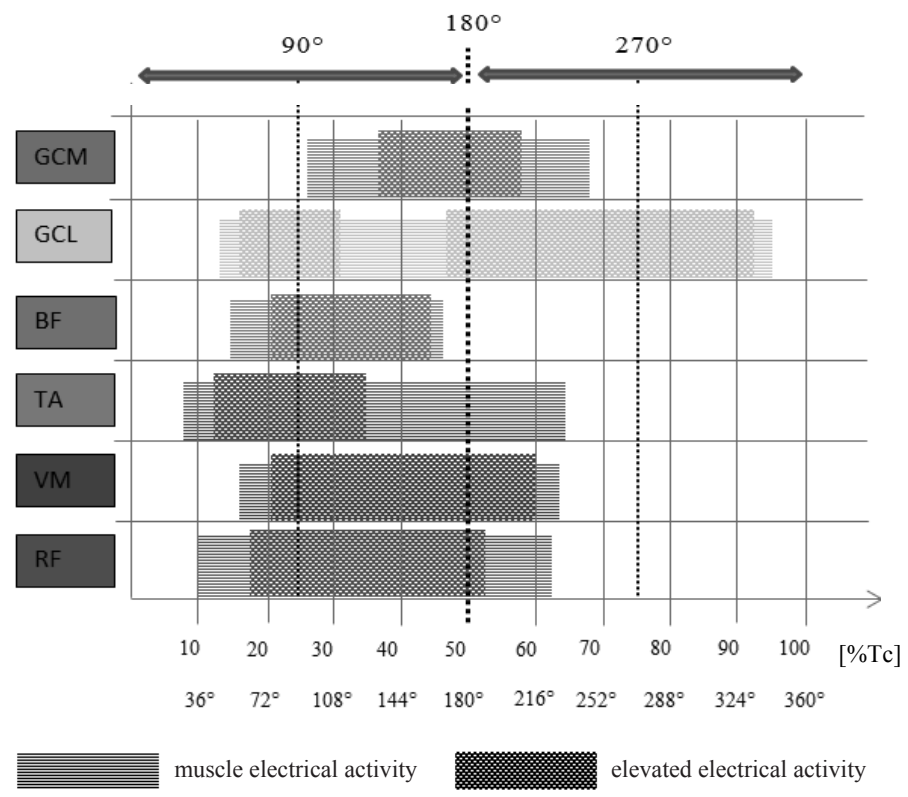

Figure 3. Bioelectrical activity of muscles in the group studied for the second variant of bicycle settings motion $\left(180^{\circ}\right)$, the periods of elevated bioelectrical activity were recorded in most of the muscles studied (GCM, BF, VM, and $\mathrm{RF}$ ). It is also remarkable that the electrical signal in the biceps femoris and tibialis anterior muscles disappeared at around half of the range of the pedalling motion.

The bioelectrical activity of the muscles of the lower limbs during pedalling on a cycle ergometer with the saddle set low and with toe clips is presented schematically in Figure 5. The figure reveals that electrical phenomena occurred first in the TA and RF muscles (before $10 \%$ of the cycle duration). In all other muscles, these phenomena were recorded noticeably later (around 20\% of the cycle duration).

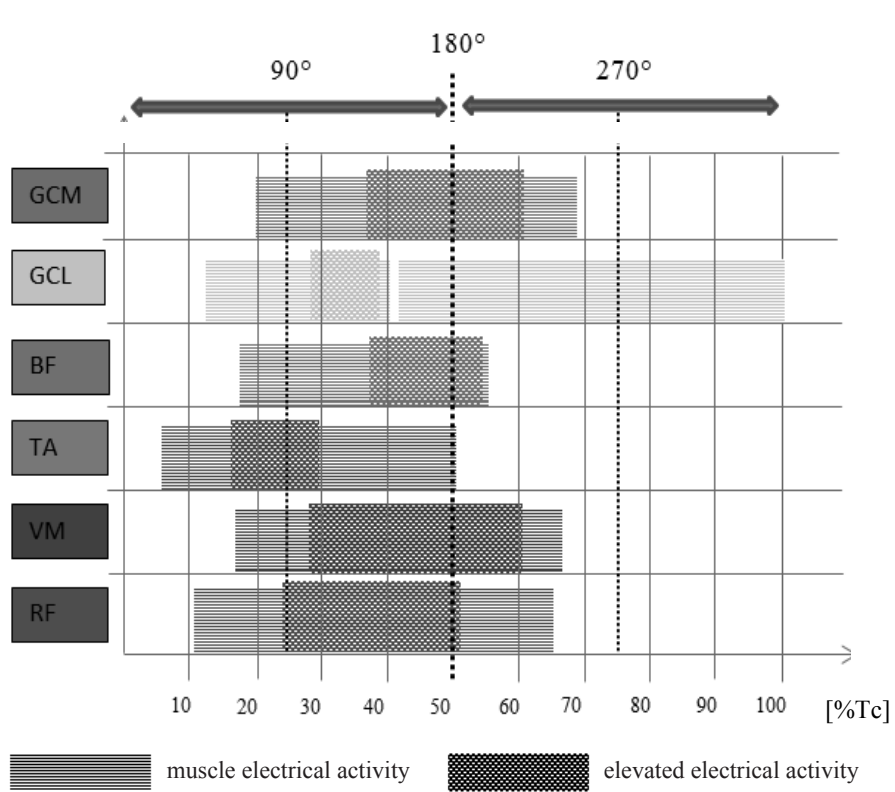

Figure 4. Bioelectrical activity of muscles in the group studied for the third variant of bicycle settings

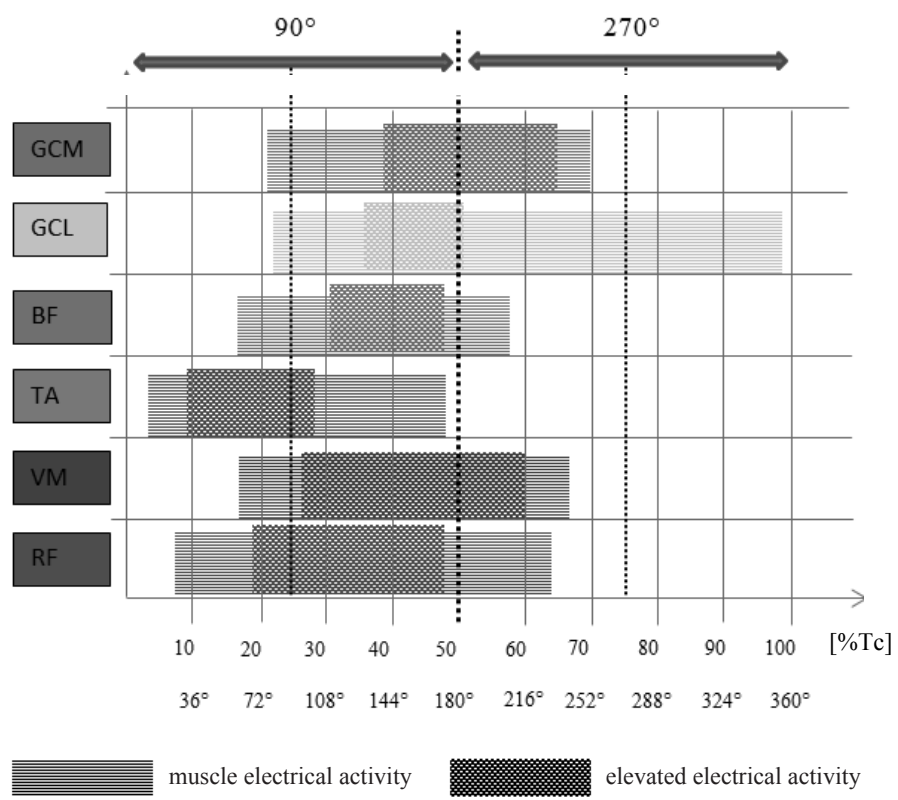

Figure 5. Bioelectrical activity of muscles in the group studied for the fourth variant of bicycle settings 
Similarly to other variants of motion (1-3), the EMG signal was sustained the longest in the gastrocnemius caput laterale (95\% of the cycle duration). The shortest time of action potential was found for the tibialis anterior muscle, where the electrical signal was very weak as early as from the middle of the crankset cycle $\left(180^{\circ}\right)$. Action potential in other muscles disappeared at around $60-70 \%$ of cycle duration (GCM, BF, VM, and RF).

The strongest EMG signal was recorded around the middle of the motion $\left(180^{\circ}\right)$ for only two muscles, namely the gastrocnemius caput laterale and vastus medialis. In the case of the four other muscles, elevated bioelectrical activity was recorded earlier (before the completion of the first half of the motion).

\section{Discussion}

According to the aim discussed above, the study evaluated the time of sustained action potential in selected muscles of the lower limbs, the order of their activation and deactivation, and the percentage share of the bioelectrical activity of individual muscles in the crankset motion (pedalling) cycle. Furthermore, the study examined the effect of saddle position and the use of toe clips on the above measures. All the variables were determined with reference to the pedalling method.

Electrical potential duration in the muscles studied was on average 0.5-0.6 s, and the differences recorded for individual pedalling variants were minimal. In absolute terms, these values are difficult to compare unequivocally with the data obtained by other authors because the time of the recording of biopotentials depends on the rotational speed of the bicycle crankset. The higher the rotational speed, the shorter the duration. This can be observed, for instance, when one compares the results obtained by Savelberg et al. [17] and Dias Lopes et al. [18]. However, when the duration of the biopotential recording was compared to the duration of a single crankset rotation, the results of this study turned out to be similar to those of several previous studies, once the findings of other authors [10, 19] were re-calculated for this purpose.

The results obtained showed that only one period of elevated bioelectrical activity of the muscles can be observed over the pedalling cycle, regardless of the gender of the subject. This leads to the conclusion that each muscle group has its specific time of elevated activity during the time of a single crankset rotation cycle. This finding is consistent with the data documented in a previous study [20]. The data demonstrated that each pedalling cycle comprising four phases (the initial phase, the peak power phase, the "follow-up" phase, and the return phase) is characterised by elevated activity of individual muscle groups characteristic for the specific phase. In studies published both in Poland and abroad, pie charts have been used to illustrate muscle activity (understood as action potential) with respect to the angle of crankset rotation, where individual activity was emphasised for each muscle over a single cycle [11, 21, 22]. Naturally, the data presented in these studies also revealed the periods of elevated electrical potentials, but the authors did not discuss these problems. It should be emphasised that the observation of individual muscular activity is predominant in research studies. Furthermore, the findings of one study [19] showed two separate periods of activity in a single cycle for the tibialis anterior (TA) muscle. It seems that the difference in the results obtained in the studies could have been caused by an important difference in the measurement methodologies. In the present study and in the above-mentioned previous studies, measurements were performed by means of surface electrodes, whereas Chapman et al. [19] used intramuscular (needle) electrodes in measure- ments of muscle potentials. It should be emphasised that this possibility of the "double switching on" of the tibialis anterior muscle was also found in the present study. This phenomenon was observed in individual cases. However, after averaging the results in order to obtain the pattern of mean values of muscle biopotentials in the group of study participants, this pattern was modified. Bober and Zawadzki [23] argue that the recording of electrical voltage in the active muscle makes it possible to evaluate whether the muscle was excited. However, excitation does not necessarily mean that activity-induced change in muscle length occurred, i.e. the origin and insertion became closer or further to each other. The change in muscle length can be indirectly evaluated based on changes in joint angles. It can be observed that the presence of action potential alone is insufficient for the evaluation of the sign in muscle potential (negative - eccentric, positive - concentric). Furthermore, the time of biopotential presence may be not linked to the time of muscle action. This stems from the fact that a latent period is sometimes observed, and the thresholds of the excitation may be different for different muscles. Finally, electrical phenomena in the muscles depend on their functional state, and the latter varies individually. Therefore, the presence of action potential in the muscles reflects their readiness to work, whereas this state of readiness can be converted into actual activity, for instance, due to an unbalanced resultant of forces (e.g. resulting from external control of the cycle ergometer in terms of the saddle height, toe clips in the pedals, crankset resistance, or the gear ratio). The essential aspect of the current study was the evaluation of the effect of bicycle settings on the change in the bioelectrical activity pattern in the muscles of the lower limbs. The lowering of the saddle caused an increase in the percentage contribution of the electrical activity of the VM (vastus medialis) and the GCL (gastrocnemius caput laterale) muscles, whereas the contribution of the TA (tibialis anterior), the BF (biceps femoris), and the GCM (gastrocnemius caput laterale) muscles was reduced.

The results presented in the studies that examined the effect of changes in the bicycle settings on muscle function during riding demonstrate that changes occur even for insignificant adjustments in body configuration. Drozdek et al. [10] compared bioelectrical activity in the muscles of the same cyclist who pedalled in similar external conditions (external load) on a road bicycle and triathlon bicycle. It turned out that insignificant changes in position, connected with the design of these bicycles, affected the values of certain variables that described the action potential in the muscles of the lower limbs in the cycle of crankset rotation. It should be noted that the difference between the position on a road bicycle and triathlon bicycle is substantially smaller than the differences that result from changes in the conditions in the present study. Therefore, the results obtained in this study demonstrated bigger differences in the activity of individual muscles in varying forced conditions related to particular bicycle settings. The above problems, however, need to be further researched since, as demonstrated in the literature review presented in previous studies [24, 25], changes in the cyclist's body position affect changes in the value of electrical biopotentials in the muscles. This may be caused by the fact that a reduction in this value does not have to be linked to a reduction in mechanical muscle activity [17] as this activity can be related to the optimisation of movement technique induced by training. Therefore, it is likely that the selection of the study group may be of essential importance to the problem discussed.

Contrary to the initial assumptions, the use of toe clips in the pedals did not have a noticeable important effect on the 
duration of electrical activity in muscles. However, it should be noted that an insignificant reduction in activity was observed, particularly in the case of the BF and TA during pedalling without toe clips. All the people who participated in the study had not participated in professional cycling sports before and did not use any specific pedalling technique. This seems to have essential importance in light of technological progress in bicycle design, particularly concerning the solutions related to foot fixation to the bicycle pedal, which was mentioned in the Introduction section. The group selection method used in the study allowed for obtaining results which can be regarded as representative for an average patient of physiotherapy surgeries. A reduction in electromyographic activity during riding without toe clips was also documented by other authors [26]. It is worth noting that this observation seems to be logical, since toe clips offer additional support for the lower limbs that allows the TA muscle (the main foot extensor) and the BF muscle (the knee joint flexor) to work during the phase of the knee bend (upward pedal motion).

Interestingly, regardless of the position of the cycle ergometer, the action potential in the gastrocnemius caput laterale (GCL) was recorded for the longest time, whereas the potential in the vastus medialis was the shortest. A previous study [27] allows for a positive verification of these observations; the authors of that study compared the electrical activity of the muscles for various angles of a ground slope $(4 \%, 7 \%, 10 \%)$, and it transpired that the muscle which performed the least work was the vastus medialis, whereas the muscle with the highest percentage contribution to the cycle was the gastrocnemius muscle. Chapman et al. [19] examined cyclists and found that the gastrocnemius caput laterale was the most active muscle in all the study participants. Furthermore, the low activity of the vastus medialis muscle is likely to be caused by the fact that the process of knee extension also involves the work of four heads of the quadriceps femoris muscle. It should also be noted that the first phase of pedalling, where the lower limb is extended in the knee joint, occurs in the direction of gravity, which supports the work of the extensors of this joint. The literature also shows that cycling substantially engages the vastus lateralis muscle [7], which might cause lower activity of the VM muscle. The longest time of the presence of the action potential of the GCL muscle is likely to be caused by the foot position on the pedal. The crankset is moved with the pressure of the forefoot, with the main movement of the knee joint being plantar flexion [7] driven by the gastrocnemius muscle. Consequently, changes in the quality of the EMG signal in individual muscles during pedalling can be expected. However, the analysis of variance did not provide evidence confirming that the differences found in the study were significant, which noticeably affects the results obtained. The results of the present study clearly show that, due to the opportunity for the adjustment of the settings, bicycles are useful and valuable pieces of equipment that should be used in rehabilitation. It should also be added that, through the individual adjustment of bicycle settings, physiotherapists can extend the range of available methods to stimulate the patient's locomotor system. Using a conventional bicycle (outside of the laboratory) is a pleasurable of maintaining physical fitness. In their study, Bertucci et al. [28] demonstrated that the muscular work of the lower limbs on a cycle ergometer does not significantly differ from the work on an outdoor bicycle. Therefore, in justified cases (under the supervision of a therapist), a stationary bicycle can be replaced with a bicycle for outdoor use that almost every person has.

\section{Objectives for further research}

Undoubtedly, the examinations carried out did not provide a full picture of the problems discussed in the study. However, the findings make it possible to formulate the objectives for similar measurements in the future. It seems that important findings can be expected from examinations conducted in a much bigger group. Furthermore, the changes in pedalling frequency and power developed by the lower limb muscles should be more extensive in future studies (the frequency of movements and muscle power remained unchanged in this study, with $1 \mathrm{~Hz}$ and $100 \mathrm{~W}$, respectively).

\section{Conclusions}

The analysis of the results obtained in this study leads to the following conclusions:

1. Only one period of elevated electrical activity in the muscles of the lower limbs is observed in the pedalling cycle.

2. The change in saddle height for the cycle ergometer and the use of toe clips in the pedals affect the changes in the bioelectrical activity profile in the muscles. The adjustment of saddle height affects the duration of potentials more noticeably than the use of toe clips.

3. Regardless of the variant of cycle ergometer settings, action potential was recorded for the longest time in the gastrocnemius caput laterale (GCL), whereas the potential in the vastus medialis was the shortest.

4. The order of the appearance and disappearance of action potential is the same for the same saddle height and is not affected by the use of toe clips.

5. Except for the gastrocnemius caput laterale (GCL), the action potential of other muscles of the lower limb is reduced after 60 to $70 \%$ of the cycle duration, which corresponds to the angle of crankset rotation of around $220-250^{\circ}$.

\section{Literature}

1. Fonda B., Sarabon N. (2010). Inter-muscular coordination during uphill cycling in a seated position: A pilot study. Kinesiologia Slovenica 16(1-2), 10-15.

2. Faria E., Parker D., Faria I. (2005). The science of cycling: Factors affecting performance - part 2. Sports Medicine 35(4), 313-337.

3. Gamez J., Zarzoso M., Raventos A., Valero M., Alcantara E., Lopez A. et al. (2008). Determination of the optimal saddle height for leisure cycling. The Engineering of Sport 7, 255260. DOI: $10.1007 / 978-2-287-99056-4$ _31.

4. Horscroft R., Davidson C., McDaniel J., Wagner B., Martin J. (2003). Effects of saddle height on joint power distribution. Medicine and Science in Sports and Exercise 35(5), 16.

5. Wanich T., Hodgkins C., Columbier J., Muraski E., Kennedy J. (2007). Cycling injuries of the lower extremity. Journal of the American Academy of Orthopaedic Surgeons 15(12), 748-756.

6. Hansen E., Smith G. (2009). Factors affecting cadence choice during submaximal cycling and cadence influence on performance. International Journal of Sports Physiology and Performance 4, 3-17.

7. Li L., Caldwell G. (1998). Muscle coordination in cycling: Effect of surface incline and posture. Journal of Applied Physiology 85(3), 927-934.

8. Cordova A., Latasa I., Seco J., Villa G., Rodriguez-Falces J. (2014). Physiological responses during cycling with oval 
chainrings (Q-Ring) and circular chainrings. Journal of Sports Science and Medicine 13, 410-416.

9. Costan R., Pantea C. (2010). Electromyography assessment of muscles involved in a pedal cycle. Timisoara Physical Education and Rehabilitation Journal 2(4), 29-36.

10. Drozdek R., Winiarski S., Siemieński A. (2014). Differences in the bioelectrical activity of the major muscle groups of the lower limb using road or triathlon position. In C. Urbanik, A. Mastalerz, D. Iwańska (ed.), Selected problems of biomechanics of sport and rehabilitation (pp. 12-20). Warszawa: AWF Warszawa.

11. Dorel S., Couturier A., Hug F. (2009). Influence of different racing positions on mechanical and electromyographic patterns during pedaling. Scandinavian Journal of Medicine E Science in Sports 19, 44-54. DOI: 10.1111/j.16000838.2007.00765.x.

12. Burke E. (1994). Proper fit of the bicycle. Clinics in Sports Medicine 13(1), 1-14.

13. de Vey Mestdagh K. (1998). Personal perspective: In search of an optimum cycling posture. Applied Ergonomics 29(5), 325-334.

14. Silberman M., Webner D., Collina S., Shiple B. (2005). Road bicycle fit. Clinical Journal of Sport Medicine 15(4), 271-276.

15. Ericson M., Nisell R. (1987). Patellofemoral joint forces during ergometric cycling. Physical Therapy 67, 1365-1369.

16. Ericson M. (1986). On the biomechanics of cycling. A study of joint and muscle load during exercise on the bicycle ergometer. Scandinavian Journal of Rehabilitation Medicine $16,1-43$.

17. Savelberg H., Van de Port I., Willems P. (2003). Body configuration in cycling affects muscle recruitment and movement pattern. Journal of Applied Biomechanics 19, 310-324.

18. Dias Lopes A., Alouche S., Hakansson N., Cohen M. (2014). Electromyography during pedaling on upright and recum- bent ergometer. The International Journal of Sports Physical Therapy 9(1), 76-81.

19. Chapman A., Vicenzino B., Blanch P., Knox J., Hodges P. (2006). Leg muscle recruitment in highly trained cyclists. Journal of Sports Sciences 47(2), 115-124. DOI: 10.1080/02640410500131159.

20. Kosielski P. (2012). Anatomy of pedalling. Retrieved September 10, 2014, from www.medicycycling.eu. [in Polish]

21. Fonda B., Sarabon N. (2010). Biomechanics of cycling: Literature review. Sport Science Review 19(1-2), 131-163. DOI: 10.2478/v10237-011-0012-0.

22. Jorge M., Hull M. (1986). Analysis of EMG measurements during bicycle pedaling. Journal of Biomechanics 19(9), 683-694.

23. Bober T., Zawadzki J. (2003). Biomechanics of the human locomotor system. Wrocław: BIK. [in Polish]

24. Bini R., Diefenthaeler F. (2009). Mechanical work and coordinative pattern of cycling: A literature review. Kinesiology 41(1), 25-39. UDC 796.61:796.015.62:531.6.

25. Bini R., Hume P., Lanferdini F., Vaz M. (2014). Effects of body positions on the saddle on pedaling technique for cyclists and triathletes. European Journal of Sport Science 14(1), 413-420. DOI: 10.1080/17461391.2012.708792.

26. Cruz C., Bankoff A. (2001). Electromyography in cycling: Difference between clipless pedal and toe clip pedal. Electromyography and Clinical Neurophysiology 41(4), 247-52.

27. Duc S., Bertucci W., Pernin J., Grappe F. (2008). Muscular activity during uphill cycling: Effect of slope, posture, hand grip position and constrained bicycle lateral sways. Journal of Electromyography and Kinesiology 18, 116-127. DOI: 10.1016/j.jelekin.2006.09.007.

28. Bertucci W., Grappe F., Groslambert A. (2007). Laboratory versus outdoor cycling conditions: Differences in pedaling biomechanics. Journal of Applied Biomechanics 23, 87-92.

Submitted: March 23, 2017

Accepted: July 28, 2017 